\title{
Current Management of Juvenile Recurrent Parotitis
}

\author{
Johannes Zenk • Helgard Schneider • \\ Michael Koch $\cdot$ Heinrich Iro
}

Published online: 14 January 2014

(c) Springer Science+Business Media New York 2014

\begin{abstract}
Juvenile recurrent parotitis is a disease of unclear etiology. It occurs between the ages of 4 months and 15 years, but is usually self-limiting with puberty. Clinical features consist of unilateral, occasionally bilateral, episodic swelling of the parotid with pain, fever, and hyposecretion by the affected salivary gland. Numerous theories have been proposed for its pathogenesis, with almost as many diagnostic and therapeutic approaches. As the cause is still unexplained, various types of treatment have been tried over the years. This article discusses the most commonly used conservative and surgical treatment options of the last decades.
\end{abstract}

Keywords Juvenile recurrent parotitis - Conservative therapeutic options $\cdot$ Surgical treatment $\cdot$ Sialendoscopy

\section{Introduction}

Juvenile recurrent parotitis (JRP) is an inflammatory disease of the salivary glands of unknown etiology. It is characterized by recurrent unilateral or bilateral swelling of the parotid gland with pain, redness, occasionally fever and

\footnotetext{
J. Zenk $(\bowtie) \cdot$ H. Schneider $\cdot$ M. Koch · H. Iro

Department of Otorhinolaryngology, Head and Neck Surgery,

University of Erlangen-Nuremberg Medical School,

Waldstrasse 1, 91054 Erlangen, Germany

e-mail: johannes.zenk@uk-erlangen.de

H. Schneider

e-mail: helgard.schneider@uk-erlangen.de

M. Koch

e-mail: michael.koch@uk-erlangen.de

H. Iro

e-mail: heinrich.iro@uk-erlangen.de
}

hyposecretion by the affected gland. Flares usually last for 1 or 2 days, in rare cases for weeks; the interval between flares may even last for some years. Hyposecretion of the affected gland may also be demonstrated in these symptom-free periods, sometimes with very viscous or flocculent saliva $[1 \bullet \cdot]$. The age of onset is between 4 months and 15 years, but the disease is usually self-limiting with puberty. Boys are affected more often than girls. Apart from JRP as described here, there is an adult form of chronic recurrent parotitis, which is about ten times more common [2]. The adult form more often affects women and involves the distal salivary duct, which appears stenotic or dilated. In contrast, the classic pathological change in JRP is duct ectasia of the intraglandular duct $[1 \bullet \bullet$. Various imaging techniques often demonstrate duct pathology on the asymptomatic side as well, although it is usually less pronounced [3, 4••]. In histological examinations, JRP presents itself in three stages: stage I with periductal lymphocytic infiltration; stage II showing the formation of lymphatic follicles; stage III, which is seldom seen, with lymphatic transformation of the gland [5]. Despite the fact that JRP is very rare, it is still one of the most common salivary gland diseases of childhood after mumps.

The etiology of the disease is disputed to this day and remains the subject of many discussions, although the theory of multifactorial etiology is generally preferred today. As early as 1945, Hamilton Bailey proposed the presence of a congenital abnormality. He drew a parallel to bronchiectasis and suggested a congenital dilatation of the salivary duct system, which would be predisposed to infection [6]. On the other hand, in the early 1970s, Hemenway thought the cause to be a lymphocytic infiltration of the duct walls with loss of acini, resulting in secondary bacterial infection and inflammatory changes of the gland structure [7]. An association with viral infections such as 
mumps has not been made [3]. Bacterial infection is also controversial. As pus is only very rarely found, the administration of antibiotics in the absence of a bacterial infection is disputed $[8 \cdot \bullet$. Congenital changes in the duct system $[9,10,11 \bullet, 12,13]$, autoimmune disease [9, 14], autosomal dominant inheritance [15], immune deficiency [3, 16, 17], and disrupted enzyme activity [18] have all been discussed. In 2004, Nahlieli proposed a further hypothesis based on the endoscopic picture of the ducts during sialendoscopy. The underlying cause here is a clearly visible reduction in the blood vessels, which has a detrimental effect on salivary secretion and results in chronic inflammation [19•].

Apart from the clinical findings suggesting the diagnosis, the investigation of choice is a B-mode ultrasound scan. The classic image shows hypoechoic, loosely structured, cotton-wool-like tissue corresponding to lymph nodes and the typical sialectasis [1••]. Although the symptoms are different, Sjögren's syndrome and sarcoidosis may present a similar ultrasound picture [20, 21••]. Ultrasound scanning, which is non-invasive and does not expose the patient to X-rays, is a very good alternative to sialography. Pathognomonic sialographic findings demonstrate the characteristic sialectasis as an accumulation of contrast medium in the dilated intraparenchymal ducts [22]. The asymptomatic side may also be affected in this way, but it is not usually so pronounced [9]. Further possibilities of demonstrating the gland include magnetic resonance imaging (MRI) [12], which is more costly and may require sedation, MR sialography [13], or computed tomography, which is not often used today because of the radiation exposure. Sialendoscopy is another possible diagnostic procedure. In this endoscopic visualization of the salivary glands, the typical findings in the salivary ducts are a whitish surface with an obvious reduction in the commonly visible ductal wall vessels $[1 \bullet \bullet, 19 \bullet]$.

\section{Therapeutic Options}

The still unexplained cause of JRP has stimulated a great deal of discussion on possible therapeutic approaches over the years, although few of the treatment options developed have proved their worth in the long term. A distinction can be made between conservative management and surgical treatment as well as intraductal injection therapy and sialendoscopy.

\section{Conservative Management}

The aim of treating an acute flare is to relieve the symptoms and prevent parenchymal damage. Antibiotics are currently the most commonly used treatment for the flare, often combined with sialogogues and gland massage. If there is no evidence of the presence of staphylococci, penicillins, cephalosporins, or macrolide antibiotics may be prescribed; as a rule, however, aminopenicillins are used in combination with $\beta$-lactamase inhibitors $[1 \bullet \bullet, 9]$. The effects of sialogogues and gland massage in maintaining the saliva flow should not be underestimated. One big advantage of antibiotics is certainly the simplicity of treatment. Even so, their use must be regarded critically, particularly in this age of increasing multiresistant organisms. Watkin and Hobsley [23] studied the benefits of purely conservative management, i.e., antibiotic therapy and analgesia for acute flares. Over a follow-up period of 5 years, symptoms clearly improved in $64 \%$ of the 22 children treated [23]. Some authors share their view that a self-limiting disease should be treated conservatively in the first instance [23, 24]. This approach should not, however, ignore the fact that the patients sometimes suffer severely from the condition. Even though the studies found symptomatic improvement with antibiotic therapy, whether JRP is truly a bacterial infection is still disputed. In 2004 Baurmash investigated the secretion-mucopus-from the affected gland in an adult with chronic recurrent parotitis. $\mathrm{He}$ demonstrated a considerably increased concentration of serum proteins; however, the myeloperoxidases used as leucocyte markers were demonstrated only on the first day of the illness. This finding indicates that the condition is not a bacterial infection, but rather an inflammation of the tissues. Even though the study did not investigate saliva from a child, the use of antibiotics in recurrent parotitis is debatable $[8 \cdot \bullet]$.

Maier et al. [25] viewed the cause of the disease as increased activity of the kallikrein-kinin system in the gland. Based on this hypothesis, they treated seven adults intravenously with the kallikrein inhibitor aprotinin. Apart from the relief of symptoms, six patients showed a reduction in kallikrein activity, which had been raised in all patients before the intervention [25].

A further conservative treatment option recommended in the 1970s, but now considered obsolete, was radiotherapy of the gland. Glasenapp reported symptomatic improvement and the absence of any damage to the structure of the gland following irradiation. The five cases described were free of recurrence 1 year after a dose of up to $600 \mathrm{R}(\mathrm{R}=\mathrm{Rönt}-$ gen, legacy unit for the radiation dose) given over a period of 2-4 weeks. Side effects such as xerostomia did not occur, and no functional disturbance was found in the scintigraphic follow-up [26]. Despite these findings, radiotherapy is today considered obsolete in the light of possible sequelae, such as the increased risk of a second malignancy, xerostomia, and possible osteomyelitis. 
Surgical Treatment

Neurectomy of the tympanic nerve, described by Daud in 1995 [27], has to be mentioned as a possible surgical procedure in recurrent parotitis. Division of the secretory fibers of the tympanic nerve should reduce the gland's secretion. Seven out of ten adults had a clear improvement in their symptoms [27]. The method remains controversial because of its general lack of success and relative invasiveness [28, 29]; in particular, it is not recommended for children. A further surgical option for JRP is parotidectomy, which was considered controversial even in the 1950s. Eddey and McKenzie favored this option in severe cases [30]; in 1961, Beahrs et al. [31] published a study with 29 patients (aged 4-81 years) who were successfully treated surgically without any nerve paralysis. Despite these results, parotidectomy, as a relatively invasive procedure with an inherent risk of facial nerve paralysis, is today seen only as a last resort. As an alternative to complete parotidectomy, Diamant et al. [32] proposed ligation of the parotid duct leading to atrophy of the gland. A study in 1963 found that 11 of 18 patients (aged 3-78) had no symptoms following the procedure. This concept is still recommended in the current literature as an option preferable to parotidectomy in refractory cases. However, even under optimal conditions, the success rate of this treatment is only $50 \%$, because of ductal rupture, failed ligature, or the formation of sialocoeles [8••].

\section{Intraductal Injection Therapy and Sialendoscopy}

In an animal study, Rettinger et al. [33] occluded the parotid duct using an alcoholic prolamine emulsion (Ethib$\left.1 c^{\circledR}\right)$, with the aim of inducing atrophy of the parotid parenchyma. This principle has similar effects to duct ligation, but without the danger of salivary cysts or a failed ligature. In the case of ductal rupture, the injected material was encapsulated [33]. Both duct ligation and occlusion with Ethibloc ${ }^{\circledR}$ injection carry the risk of abscess formation, which would result in much more difficult operating conditions should surgery subsequently be required $[1 \bullet \cdot]$. Wang et al. [34] described another approach to duct occlusion. In 1998, they reported on 16 patients, whose symptoms improved considerably after gland atrophy induced by the intraductal injection of methyl violet. No side effects were reported [34]. However, as already described for Ethibloc $^{\circledR}$ injections, the risk of abscess formation following ductal occlusion of the gland also has to be considered with this procedure. In 1992, Bowling injected tetracycline into the parotid duct. Based on the therapeutic use of tetracyclines to induce fibrosis in intrapleural sclerotherapy, he treated the ducts of ten rabbits in this way. The cytotoxic effects of this antibiotic caused inflammation, fibrosis, and finally atrophy of the gland tissue [28]. Apart from the risk of abscesses, the administration of tetracyclines to children is obsolete.

Another method of treatment for recurrent parotitis was discovered by Galili et al. [11 ••] in the course their examinations, using sialography. Beside the diagnostic purpose, they found a therapeutic effect from irrigation with contrast medium. In addition to the improvement in symptoms, $86 \%$ of the 22 children treated in this way had a notable reduction in the frequency of flares [11•*]. Based on these results, the therapeutic benefits of sialendoscopy, which first became popular in the 1990s, became established. Treatment success of this initially purely diagnostic procedure seemed to be due to the irrigation, e.g., with cortisone solution, and the associated removal of deposits from the parotid duct [8••]. The beneficial effects of using tissue irrigation alone were also highlighted by Antoniades et al. [35]. They demonstrated a clear improvement in symptoms on intraductal irrigation with either penicillin or pure normal saline solution [35].

This effect, seen in sialendoscopy, has been discussed by several authors in recent years (Table 1). Nahlieli et al. [19•] treated 26 children with bilateral sialendoscopy, including cortisone irrigation. Additional balloon dilatation was carried out as part of the procedure if duct stenosis was found. Over a follow-up period of 4-36 months, only two children had recurrence on the contralateral side, for which sialendoscopy was again performed [19•]. Shacham et al. [4••] carried out this long-term study over a period of 14 years and presented results from 70 children, 56 of whom were symptom-free after a follow-up period of 6-36 months. The other 14 children were monitored closely for a year. Sialendoscopy was repeated in five cases because of the severity of the symptoms, after which only one boy had persistent problems $[4 \cdot \bullet]$. Here, it should be remembered that all of the children in these two studies had been examined using bilateral sialography prior to the intervention. MartinsCarvalho et al. [36॰] treated 18 children with sialendoscopy and cortisone irrigation; only four had postinterventional recurrence in the 4-24 month follow-up. Sialendoscopy was performed three times in three of these four cases. Balloon dilatation was also carried out here whenever there was duct stenosis [36•]. Quenin [37•] treated ten children with sialendoscopy and cortisone irrigation as well as balloon dilatation if necessary. Nine of the ten patients had no symptoms after a follow-up of 2-24 months; in one child the symptoms recurred, and the sialendoscopy was repeated. This author also reported that two patients developed symptoms again during the course of the study - but on the contralateral side that had not been treated [37•]. Capaccio et al. [38•] treated 14 children with sialendoscopy including cortisone and antibiotic (rifamycin) irrigation. Only five children had a recurrence in the 12-55 months of follow-up. Three of these five children underwent repeat sialendoscopy, after at least two flares had occurred within 1 year [38•]. 
These results demonstrated the therapeutic success of sialendoscopy, which we ourselves were able to confirm in a study. In the first retrospective comparative study on 36 children, Schneider et al. [39••] compared purely conservative management, i.e., observation and antibiotic therapy in acute exacerbations (21 patients), with the possibility of sialendoscopy with cortisone irrigation in the non-acute stage (15 patients). Acute flares were primarily always treated with antibiotics. After explaining further therapeutic options to the patients and/or parents, they were given the choice of the two therapeutic options. Sialendoscopy was performed with a semiflexible endoscope (Erlangen type, Karl Storz) with an external diameter of 0.8-1.1 mm. The duct system was dilated by continuous irrigation until the secondary and tertiary ducts could be seen (Fig. 1). The irrigation solution consisted of $100 \mathrm{mg}$ prednisolone dissolved in $50 \mathrm{ml}$ sodium chloride. Antibiotic therapy with an aminopenicillin, possibly combined with a $\beta$-lactamase inhibitor, was given peri- and postoperatively for 5 days. The only side effect observed was transient postinterventional swelling in the parotid region. In the follow-up period of 2-56 months (median: 12 months), 9 of the 15 children treated endoscopically had a recurrence, although

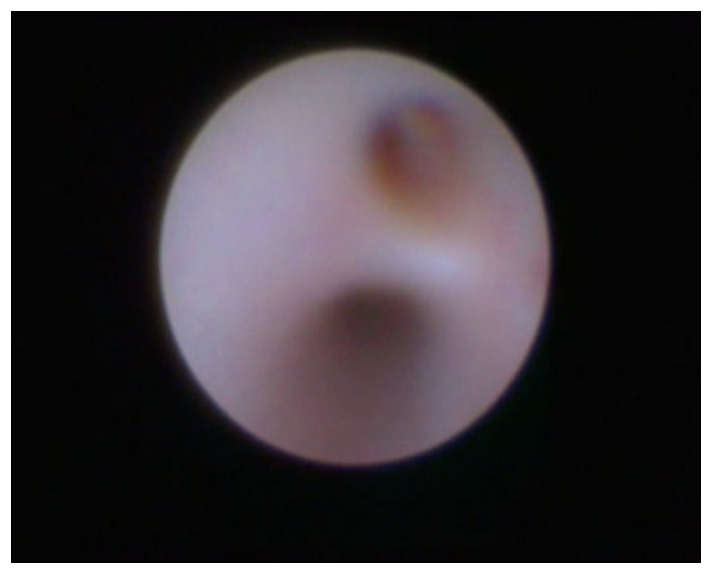

Fig. 1 Sialendoscopy: whitish thickening of the duct walls with loss of vessels in the hilum of the parotid gland it was usually much less severe. Only two of these children were treated more than once by sialendoscopy, on the express wishes of their parents. Antibiotic therapy in the non-sialendoscopy group was given orally or intravenously, depending on the severity of the symptoms. The children and/or their parents made a retrospective estimation of the frequency of pre- and post-therapeutic flares and the severity of the pain. Both of the procedures mentioned reduced not only the posttherapeutic pain, but also the frequency of the flares. Direct comparison showed two similarly effective treatment options. There are, however, clear advantages to sialendoscopy. One of these is certainly the great diagnostic value of the technique [39••]. Studies attribute more diagnostic value to endoscopy than to ultrasound scanning [36•,37•]. In the study by Schneider et al. [39••], it was particularly notable that the median follow-up was considerably shorter in the endoscopically treated group, although the outcome was equally good. This may indicate that the duration of treatment is shorter with sialendoscopy, but further prospective studies are certainly needed for confirmation. It should be remembered that, with just two exceptions, sialendoscopy was performed only once in each patient [39*0]. In line with the hypothesis proposed by Nahlieli-that the lack of vessels seen on endoscopy is responsible for the disrupted transport of saliva and hence for chronic inflammation [19•]—sialendoscopy including irrigation with anti-inflammatory cortisone is, in our opinion, an excellent method for treating JRP [39••]. We are planning prospective studies with larger numbers of patients. However, despite its advantages, sialendoscopy is still an invasive procedure that has to be carried out under a local or general anaesthetic, depending on the age and compliance of the child.

\section{Conclusions}

Summarizing the treatment options described in the literature, it is our opinion that, at the present time, the conservative management of JRP consists of antibiotic therapy

Table 1 Comparison of studies on sialendoscopy with cortisone irrigation

Results of sialendoscopy with cortisone irrigation

\begin{tabular}{|c|c|c|c|c|}
\hline & $\begin{array}{l}\text { Number of children } \\
\text { treated }\end{array}$ & $\begin{array}{l}\text { Follow-up period } \\
\text { (months) }\end{array}$ & $\begin{array}{l}\text { Number of flares } \\
\text { after treatment }\end{array}$ & $\begin{array}{l}\text { Children needing } \\
\text { repeat endoscopy }\end{array}$ \\
\hline Nahlieli et al. $[19 \bullet]$ & 26 & $4-36$ & 2 (contralateral side) & 2 \\
\hline Shacham et al. $[4 \bullet \bullet]$ & 70 & $6-36$ & 14 & 5 \\
\hline Martins-Carvalho et al. [36•] & 18 & $4-24$ & 4 & 3 \\
\hline Quenin et al. $[37 \bullet]$ & 10 & $2-24$ & 1 & 1 \\
\hline Capaccio et al. [38•] & 14 & $12-55$ & 5 & 3 \\
\hline Schneider et al. $[39 \bullet \bullet]$ & 15 & 2-65 (Median: 12) & 9 & 2 \\
\hline
\end{tabular}


of the acute flare alone or the possibility of sialendoscopy with cortisone irrigation in the non-acute phase of the flare. In retrospect, the two methods of treatment have shown themselves to be of similar value. Further prospective studies are needed to clarify whether the advantages of endoscopic therapy mentioned previously should lead to it becoming the conservative treatment of choice.

Our therapeutic strategy is based on the frequency of flares and the severity of symptoms. With only infrequent flares and mild pain, we recommend antibiotic therapy of the acute flare together with sialagogues and gland massage. When flares recur frequently and symptoms are pronounced, we prefer to carry out sialendoscopy with cortisone irrigation, after we have discussed this option thoroughly with the parents. If the symptoms are very severe and no improvement is seen with conservative management, then parotidectomy has to be mentioned as a surgical procedure. However, because of its invasive nature, the risk of facial paralysis and the fact that children usually grow out of the condition with puberty, this option should be viewed very critically and used only in rare exceptions.

\section{Compliance with Ethics Guidelines}

Conflict of Interest Johannes Zenk, Helgard Schneider, Michael Koch, and Heinrich Iro declare no conflicts of interest.

Human and Animal Rights and Informed Consent This article does not contain any studies with human or animal subjects performed by the authors.

\section{References}

Recently published papers that are of particular interest have been highlighted as:

- Of importance

•• Of major importance

1. • Zenk J, Koch M, Klintworth N, Iro H. The chronic recurrent parotitis. HNO 2010;58:237-43. Good overview of the etiology, diagnostic investigation, and treatment options of recurrent parotitis.

2. Seifert G. Aetiological and histological classification of sialadenitis. Pathologica. 1997;89:7-17.

3. Konno A, Ito E. A study on the pathogenesis of recurrent parotitis in childhood. Ann Otol Rhinol Laryngol Suppl. 1979;88:1-20.

4. • Shacham R, Droma EB, London D, Bar T, Nahlieli O. Longterm experience with endoscopic diagnosis and treatment of juvenile recurrent parotitis. J Oral Maxillofac Surg 2009;67:162-7. Large-scale long-term study on sialendoscopy, presenting its therapeutic success.

5. Ussmüller J, Donath K. Clinical, histopathologic and immunohistochemical studies of chronic sialectatic parotitis in childhood and adolescence. Klin Padiatr. 1999;211:165-71.
6. Bailey H. Congenital parotid sialectasis. J Int Coll Surg. 1945;8:109-14.

7. Hemenway WG. Chronic punctate parotitis. The Laryngoscope. 1971;81:485-509.

8. • Baurmash HD. Chronic recurrent parotitis: A closer look at its origin, diagnosis, and management. J Oral Maxillofac Surg 2004;62:1010-18. Study analyzing the parotid secretion in recurrent parotitis. The study shows that the condition is probably not due to bacterial infection, but rather to inflammation. Therapeutic options are also discussed.

9. Ericson S, Zetterlund B, Ohman J. Recurrent parotitis and sialectasis in childhood: clinical, radiologic, immunologic, bacteriologic, and histologic study. Ann Otol Rhinol Laryngol. 1991;100:527-35.

10. Ericson S, Sjoback I. Salivary factors in children with recurrent parotitis. Part2: protein, albumin, amylase, IgA, lactoferrin, lysozyme and kallikrein concentrations. Swed Dent J. 1996;20:199-207.

11. - Galili D, Marmary Y. Juvenile recurrent parotitis: clinicoradiologic follow-up study and the beneficial effect of sialography. Oral Surg Oral Med Oral Pathol 1986;61:550-6. The study shows the therapeutic success of sialography due to contrast medium irrigation.

12. Huisman TA, Holzmann D, Nadal D. MRI of chronic recurrent parotitis in childhood. J Comput Assist Tomogr. 2001;25:269-73.

13. Wittekind C, Jungehulsing M, Fischbach R, Landwehr P. Chronic recurrent parotitis in childhood in monozygotic twins. Magnetic resonance sialography. HNO. 2000;48:221-5.

14. Friis B, Pedersen K, Schiodt M, Wiik A, Hoj L, Andersen V. Immunological studies in two children with recurrent parotitis. Acta Paediatr Scand. 1983;72:265-8.

15. Reid E, Douglas F, Crow Y, Hollmann A, Gibson J. Autosomal dominant juvenile recurrent parotitis. $\mathrm{J}$ Med Genet. 1998;35:417-9.

16. Marsman WA, Sukhai RN. Recurrent parotitis and isolated IgG3 subclass deficiency. Eur J Pediatr. 1999;158:684.

17. Fazekas T, Wiesbauer P, Schroth B, Pötschger U, Gadner H, Heitger A. Selective IgA deficiency in children with recurrent parotitis of childhood. Pediatr Infect Dis J. 2005;24:461-2.

18. Kolho KL, Saarinen R, Paju A, Stenman J, Stenman UH, Pitkaranta A. New insights into juvenile parotitis. Acta Paediatr. 2005;94:1566-70.

19. - Nahlieli O, Shacham R, Shlesinger M, Eliav E. Juvenile recurrent parotitis: a new method of diagnosis and treatment. Pediatrics 2004;114:9-12. The study presents the therapeutic success of sialendoscopy. It also offers a new theory of etiology.

20. Iro H, Bozzato A, Zenk J. Atlas of head and neck ultrasound. Stuttgart New York: Thieme Medical Publishers; 2013. p. $122-30$.

21. • Zenk J, Iro H, Klintworth N, Lell M. Diagnostic imaging in sialadenitis. Oral Maxillofac Surg Clin North Am. 2009;21(3):275-92. Good overview of various imaging procedures for sialadenitis.

22. Mandel L, Witek EL. Chronic parotitis: diagnosis and treatment. J Am Dent Assoc. 2001;132:1707-11 quiz 1727.

23. Watkin GT, Hobsley M. Natural history of patients with recurrent parotitis and punctuate sialectasis. Br J Surg. 1986;73:745-8.

24. Gerterud A, Lindvall AM, Nylen O. Follow-up study of recurrent parotitis in children. Ann Otol Rhinol Laryngol. 1988;97:341-6.

25. Maier H, Adler D, Lenarz T, Muller-Esterl W. New concepts in the treatment of chronic recurrent parotitis. Arch Otorhinolaryngol. 1985;242:321-8.

26. Glasenapp GB, Kessler L, Schmidt W, Otto HJ. Treatment of recurrent parotitis by roentgen irradiation under scintigraphic control. Z Laryngol Rhinol Otol. 1970;49:520-5.

27. Daud AS, Pahor AL. Tympanic neurectomy in the management of parotid sialectasis. J Laryngol Otol. 1995;109:1155-8. 
28. Bowling DM, Ferry G, Rauch SD, Goodman ML. Intraductal tetracycline therapy for the treatment of chronic recurrent parotitis. Ear Nose Throat J. 1994;73:262-74.

29. Leerdam CM, Martin HC, Isaacs D. Recurrent parotitis of childhood. J Paediatr Child Health. 2005;41:631-4.

30. Eddey HH, McKenzie G. Surgical treatment of recurrent parotitis. Med J of Aust. 1953;2:715-7.

31. Beahrs $\mathrm{OH}$, Devine KD, Woolner LB. Parotidectomy in the treatment of chronic sialadenitis. Am J Surg. 1961;102:760-4.

32. Diamant H, Enfors B. Treatment of chronic recurrent parotitis. Laryngoscope. 1963;75:153-60.

33. Rettinger G, Stolte M, Bäumler C. Elimination of major salivary glands by temporary medicamentous occlusion of the excretory ducts. HNO. 1981;29:294-9.

34. Wang S, Li J, Zhu X, Zhao Z, Sun T, Dong H, Zhang Y. Gland atrophy following retrograde injection of methyl violet as a treatment in chronic obstructive parotitis. Oral surg Oral Med Oral Pathol. 1998;85:276-81.

35. Antoniades D, Harrison JD, Epivatianos A, Papanayotou P. Treatment of chronic sialadenitis by intraductal penicillin or saline. J Oral Maxillofac Surg. 2004;62:431-4.
36. - Martins-Carvalho C, Plouin-Gaudon I, Quenin S, Lesniak J, Froehlich P, Marchal F, Faure F. Pediatric sialendoscopy: a 5-year experience at a single institution. Arch Otolaryngol Head NeckSurg. 2010;136:33-6. The study demonstrates the diagnostic and therapeutic success of sialendoscopy.

37. - Quenin S, Plouin-Gaudon I, Marchal F, Froehlich P, Disant F, Faure F. Juvenile recurrent parotitis: sialendoscopic approach. Arch Otolaryngol Head Neck Surg 2008;134:715-9. The study demonstrates the diagnostic and therapeutic success of sialendoscopy.

38. - Capaccio P, Sigismund PE, Luca N, Marchisio P, Pignataro L. Modern management of juvenile recurrent parotitis. J Laryngol Otol 2012;126:1254-60. Good overview of the diagnostic investigation and treatment of juvenile recurrent parotitis.

39. • Schneider H, Koch M, Künzel J, Gillespie B, Grundtner P, Iro $\mathrm{H}$, Zenk J. Juvenile recurrent parotitis- a retrospective comparison of sialendoscopy versus conservative therapy. The Laryngoscope 2013. doi: 10.1002/lary.24291. The study compares the treatment success of sialendoscopy with antibiotic therapy alone. The two methods of treatment were shown to be successful, both on an individual basis and in direct comparison. 